\title{
Long segment femoral endarterectomy with aorto bifemoral bypass grafting for advanced bilateral lower limb peripheral vascular disease in a young male
}

\author{
Suraj Wasudeo Nagre* and Abhilash J \\ Associate Professor, Department of CVTS, Grant Medical College, Mumbai \\ Senior Resident, CVTS, Grant Medical College, Mumbai
}

\begin{abstract}
The ideal re-vascularisation procedure for lower limb peripheral arterial occlusive disease is decided based on the type and extent of the lesions, patient factors and the available treatment options. Femoral endarterectomy and profundaplasty has long term patency results comparable to saphenous vein grafts in infrainguinal disease, while synthetic grafts has shown inferior results with high early and late restenosis rates. This is a case report of a relatively young male smoker with extensive atherosclerosis of lower limb arteries who underwent bilateral open femoral endarterectomy (longest recorded in the institution) and infrarenal aorto-bifemoral bypass grafting with dacron Y graft. A more complete and long segment endarterectomy of distal SFA upto popliteals is technically challenging due to the friable nature of the plaque. However, if achieved as in the above case will improve the immediate postoperative clinical outcome.
\end{abstract}

Abbreviation: CFA: common femoral artery, ATA: anterior tibial artery, PTA: posterior tibial artery, DPA: dorsal pedis artery

\section{Introduction}

The ideal re-vascularisation procedure for lower limb peripheral arterial occlusive disease is decided based on the type and extent of the lesions, patient factors and the available treatment options. Femoral endarterectomy and profundaplasty has long term patency results comparable to saphenous vein grafts in infrainguinal disease, while synthetic grafts has shown inferior results with high early and late restenosis rates. This is a case report of a relatively young male smoker with extensive atherosclerosis of lower limb arteries who underwent bilateral open femoral endarterectomy (longest recorded in the institution) and infrarenal aorto-bifemoral bypass grafting with dacron Y graft.

\section{Case history}

A 44-year-old male presented with claudication pain in both hips, thighs and legs while walking, since 2 1/2 years. At present no rest pain and absolute claudication distance is of 100 meters. No h/o breathlessness, chest pain, syncope, abdominal symptoms. Patient was a chronic smoker and tobacco chewer for around 15years, stopped since 2years. No comorbidities.

On examination: Bilateral lower limbs calf muscle wasting+, hair loss + but no gangrenous changes. Both limbs were cold on touch below knees. Both femoral, popliteal, ATA, PTA, DPA pulsations were not palpable. Radial, ulnar, carotid artery pulsations normal on both sides. Ankle brachial index was 0.6 on right side and 0.5 on left side.

Arterial doppler of both lower limbs showed absent flow in both CFA, SFA, POP artery and patchy monophasic flow in right ATA and bilateral PTA and DPA, no flow noted in left ATA.
Lower limb ct angiography s/o complete occlusion of bilateral lower limb arteries involving the bifurcation of the aorta, bilateral common femoral arteries with long segment extension upto bilateral popliteal arteries (Figure 1).

Right ATA, proximal left ATA, bilateral PTA and bilateral DPA are reformed by collaterals.

SURGERY- Patient was posted for bilateral femoral endarterectomy with aortobifemoral bypass grafting, both femorals were exposed and through a transverse arteriotomy, long segment femoral endarterectomy was done on both sides, the endarterectomy specimens measured approximately $30 \mathrm{cms}$ on right side and $20 \mathrm{cms}$ on the left with intact branching pattern corresponding to the profunda femoris branch, which is the longest recorded in the institution (Figure 2). Good back flow was achieved. Through midline laparotomy abdominal aorta exposed and aorto bifemoral bypass grafting done using a dacron $\mathrm{Y} 14 \mathrm{x} 7 \mathrm{cms}, 50 \mathrm{cms}$ graft with distal anastomosis done over the femoral arteriotomy site with 6-0 prolene suture (Figure 3). Palpable popliteal, PTA pulsations were noted bilaterally in the immediate postopt period. Patient was symptomatically better with $\mathrm{ABI}$ of 0.8 and 0.7 in left and right sides respectively 1 week postoperatively.

Postoperative doppler showed: triphasic flow till popliteal arteries bilaterally and biphasic flow in right ATA, PTA and DPA, biphasic flow in left PTA and monophasic in left ATA and DPA.

Correspondence to: Suraj Wasudeo Nagre,31, Trimurti Building, J Hospital compound, Byculla, Mumbai, Pin -400008; Tel:09967795303;E-mail:surajnagre@ yahoo.com

Key words: endarterectomy, bypass grafting, dacron graft

Received: November 20, 2017; Accepted: December 08, 2017; Published: December 12, 2017 

male

\section{Discussion}

Surgical endarterectomy has been the standard treatment for occlusive disease of the common femoral artery (CFA) for over 50 years. However isolated occlusive disease of the common femoral artery (CFA) is uncommon and tends to often be associated with aortoiliac or femoro-popliteal atherosclerosis [1]. The plaques in the region

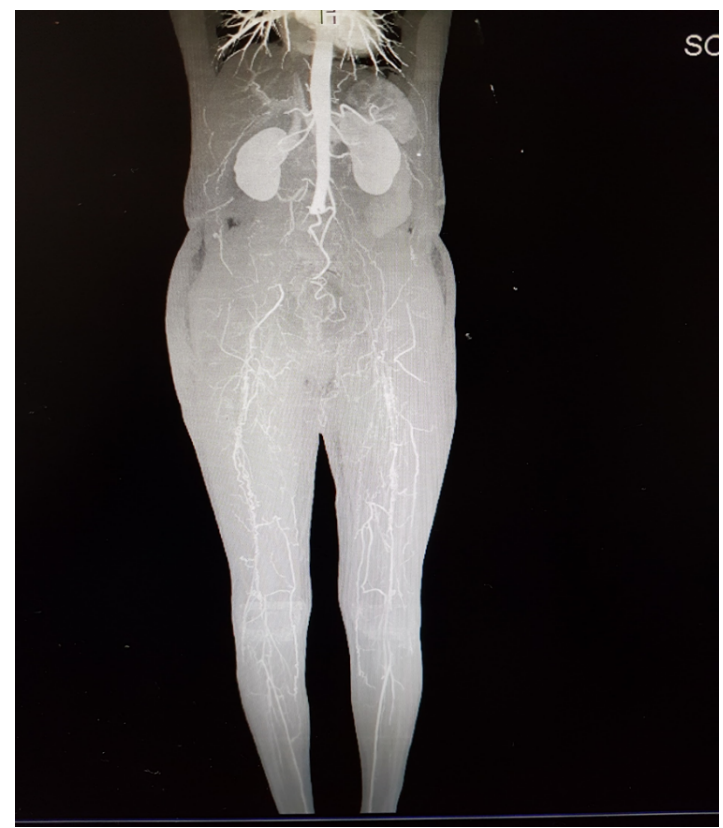

Figure 1. Lower limb CT angiography s/o complete occlusion of bilateral lower limb arteries involving the bifurcation of the aorta, bilateral common femoral arteries with long segment extension upto b/l popliteal arteries. Right ATA, proximal left ATA, bilateral PTA and bilateral DPA are reformed by collaterals.

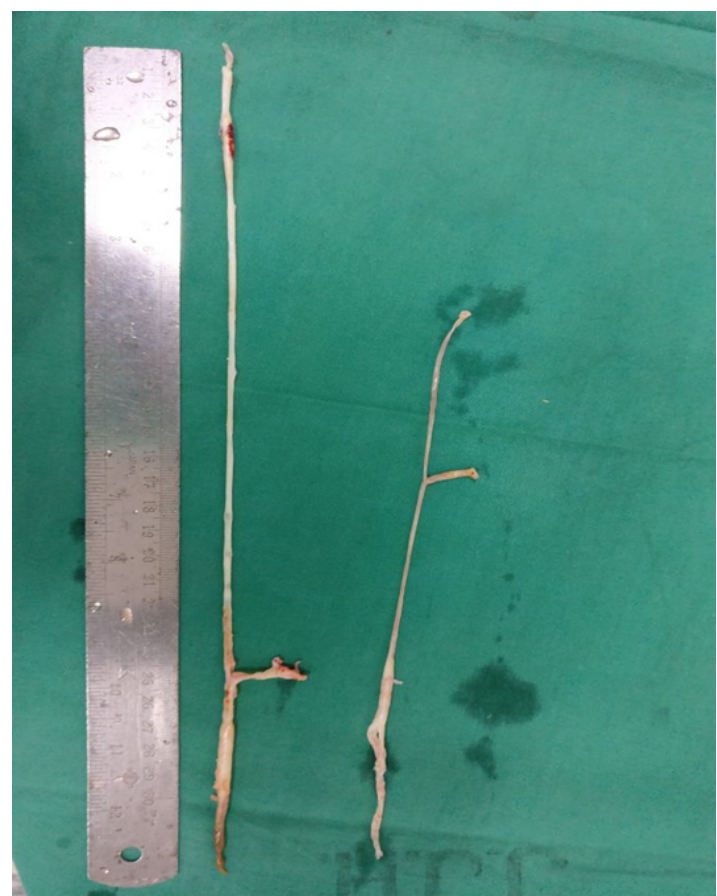

Figure 2. The endarterectomy specimens measured approximately $30 \mathrm{cms}$ on right side and $20 \mathrm{cms}$ on the left with intact branching pattern corresponding to the profunda femoris branch, which is the longest recorded in the institution

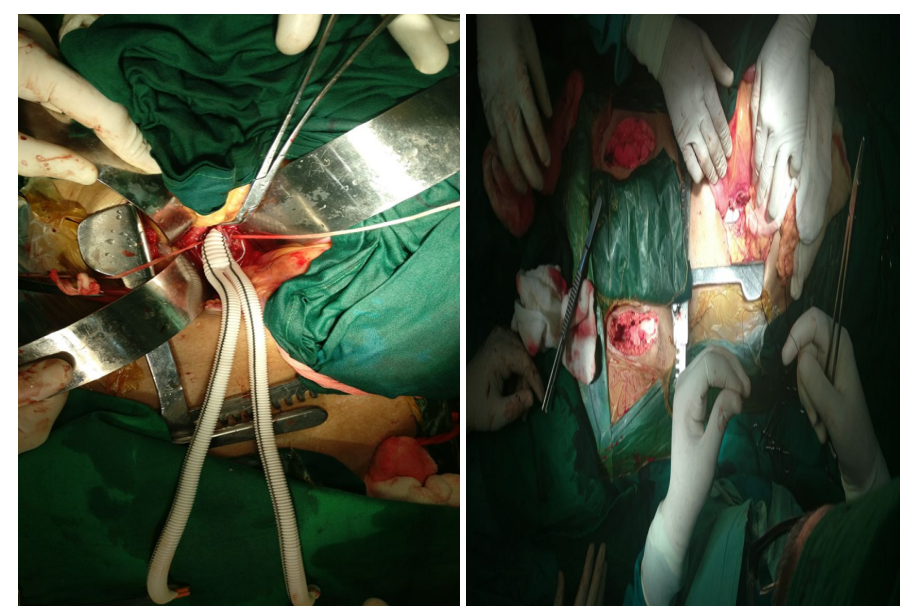

Figure 3. Aortobifemoral Dacron Y grafting

are often bulky, eccentric and highly calcified, and can be complex due to the involvement of the femoral bifurcation [2]. Moreover, the CFA is important as an access site to various endovascular procedures and a strategic region that carries the aorto-iliac artery, femorapopliteal artery and their collaterals [3]. Studies have shown that CFE (common femoral endarterecctomy) was effective for revascularization even in some chronic limb ischemia and end stage renal disease patients. CLI patients often have multi-level arterial occlusive disease, especially below-the-knee occlusive disease [4]. Patients with tissue loss or gangrene should undergo complete revascularization to the ankle as permitted by the patient's condition, although patients with claudication or rest pain might undergo CFE alone. Patients with CFA lesions and skipped SFA or below-the-knee lesions are recommended to have CFE and combined endovascular procedures while patients with complex lesions on CFA to SFA are recommended CFE with a femoropopliteal or femoro-tibial bypass [5]. Aorto-bifemoral bypass grafting is recommended for patients with lesions involving bilateral iliac arteries and CFA. Lack of studies comparing the length of endarterectomy done to the clinical outcomes achieved can be attributed to the fact that long segment common femoral endarterectomy through a small SFA arteriotomy as in the above case is seldom achieved in all cases especially in extensive long standing occlusive disease with wellformed collaterals and that in such extensive diseases outcome majorly depends on the proximal bypass grafts done along with.

\section{Conclusion}

Femoral endarterectomy as a lone procedure is seldom done nowadays and is mostly restricted to CFA and SFA segments. A more complete and long segment endarterectomy of distal SFA up to popliteal is technically challenging due to the friable nature of the plaque. However, if achieved as in the above case will improve the immediate postoperative clinical outcome.

\section{Consent}

Informed consent has been obtained.

\section{Funding}

No funding was required for this study.

\section{Conflict of interest}

No potential conflict of interest. 

male

\section{Ethical approval}

All procedures performed in studies involving human participants were in accordance with the ethical standards of the institutional and/ or national research committee and with the 1964 Helsinki declaration and its later amendments or comparable ethical standards.

\section{References}

1. Nagre Sw, Nagre MS (2015) Aortoiliac Occlusion Requiring Aortobifemoral Grafting. International Educational Scientific Research Journal.
2. Nagre Sw (2016) Femoral Endarterectomy Against Iliofemoral Bypass-Case Series. Austin J Cardiovasc Dis Atherosclerosis 3: 1027.

3. Nagre Sw (2016) Subclavian Artery Pseudoaneurysm Secondary to Accidental Stone Chip Injury and Its Excision. J Cardiovasc Med Cardiol 3: 030-031.

4. Nagre Sw (2016) Long Segment Left Anterior Descending Endarterectomy $[10 \mathrm{Cm}]$ And Its Reconstruction Using Left Internal Thoracic Artery. J Cardiovasc Med Cardiol 3:023-025.

5. Nagre Sw, Bhosle K N, Khatod A (2016) Poststab Injury Subclavian Artery Pseudoaneurysm Excision. Indian J Vasc Endovasc Surg 3:31-32.

Copyright: $@ 2017$ Nagre SW. This is an open-access article distributed under the terms of the Creative Commons Attribution License, which permits unrestricted use, distribution, and reproduction in any medium, provided the original author and source are credited. 